\title{
Orbitofrontal cortex contributes to the comparison of values underlying economic choices
}

\author{
Sébastien Ballesta ${ }^{1 \dagger}$, Weikang Shi ${ }^{1}$ and Camillo Padoa-Schioppa ${ }^{1,2,3}$
}

\begin{abstract}
Affiliations: ${ }^{1}$ Department of Neuroscience, ${ }^{2}$ Department of Economics and ${ }^{3}$ Department of Biomedical Engineering, Washington University in St. Louis, St. Louis, MO, 63110, USA.

† Present address: Laboratoire de Neurosciences Cognitives et Adaptatives (UMR 7364), Strasbourg, France. Centre de Primatologie de I'Université de Strasbourg, Niederhausbergen, France
\end{abstract}

Keywords: decision making, microstimulation, subjective value, neuroeconomics, monkey

Acknowledgments: We thank E. Bromberg-Martin, K. Conen, I. Monosov, L. Snyder, P. Werginz and members of our lab for helpful comments on previous versions of the manuscript. This research was supported by the National Institutes of Health (grants number R01DA032758 and R01-MH104494 to CPS).

Author contributions: SB and WS collected and analyzed the data; CPS supervised the project and wrote the manuscript. All the authors edited the manuscript.

Competing interests: The authors have no competing interests.

Correspondence: CPS, camillo@wustl.edu

Significance: Economic choices encompass two mental stages: values are assigned to the available offers, and a decision is made by comparing values. Previous work showed that choices are causally related to offer values encoded in the orbitofrontal cortex (OFC). Conversely, the neural underpinnings of value comparison remain poorly understood. Here we show that weak electrical stimulation of OFC selectively disrupts the decision process without affecting offer values. Hence, neurons in OFC contribute to value comparison. 
Economic choices between goods entail the computation and comparison of subjective values. Previous studies examined neuronal activity in the orbitofrontal cortex (OFC) of monkeys choosing between different types of juices. Three groups of neurons were identified: offer value cells encoding the value of individual offers, chosen juice cells encoding the identity of the chosen juice, and chosen value cells encoding the value of the chosen offer. The encoded variables capture both the input (offer value) and the output (chosen juice, chosen value) of the decision process, suggesting that values are compared within OFC. Recent work demonstrates that choices are causally linked to the activity of offer value cells. Conversely, the hypothesis that OFC contributes to value comparison has not been confirmed. Here we show that weak electrical stimulation of OFC specifically disrupts value comparison without altering offer values. This result implies that neuronal populations in OFC participate in the decision process. 


\section{Introduction}

Recent work demonstrated that offer values encoded in OFC are causal to choices (Ballesta et al., 2020). In contrast, where in the brain and how exactly values are compared to make a decision remains an open question. Several hypotheses have been put forth. When monkeys choose between goods, different groups of cells in OFC encode the choice input (offer values) and the choice outcome (chosen good, chosen value) (Padoa-Schioppa and Assad, 2006; Pastor-Bernier et al., 2019), suggesting that the cell groups identified in this area constitute the building blocks of a decision circuit. Experimental findings (Padoa-Schioppa, 2013; Rich and Wallis, 2016) and computational models (Rustichini and Padoa-Schioppa, 2015; Song et al., 2017; Zhang et al., 2018) support this hypothesis, but the evidence remains correlative. Other studies suggested that economic decisions take place in motor systems (Glimcher et al., 2005), through distributed processes (Cisek, 2012; Hunt and Hayden, 2017), through shifts of visual attention (Krajbich et al., 2010), by integrating hippocampal signals (Bakkour et al., 2019), or without the explicit comparison of values (Hayden and Moreno-Bote, 2018). However, none of these proposals has been validated by causal evidence. The present study specifically examined whether OFC contributes to value comparison.

We introduce a paradigm to assess whether a neural population contributes to a decision. At the neural level, a binary decision is ultimately a comparison between two neural signals. For example, in the random dot task used to study the visual perception of motion (Salzman et al., 1990), the decision is a comparison between two neural signals representing motion in the two valid directions. Conversely, in economic choices, the decision is a comparison between two neural signals representing the offer values. In general, the signals to be compared are the input of the decision process. Now consider an experiment in which altering the activity of a neuronal population induces higher choice variability (shallower psychometric curves). In principle, an increase in choice variability may be due (a) to noisier (more ambiguous) input signals, (b) to a noisier decision process, or (c) to the disruption of the subsequent motor response. If one can exclude (a) that the manipulation makes input signals more noisy and (c) that it disrupts motor planning, one may conclude (b) that the neural population participates in the decision process. We now consider three previous results in this light.

First, classic studies showed that low-current stimulation $(\geq 10 \mu \mathrm{A})$ of the middle temporal area biased perceptual decisions (Salzman et al., 1990; Salzman et al., 1992). Furthermore, highcurrent stimulation $(\geq 40 \mu \mathrm{A})$ increased choice variability (Murasugi et al., 1993). The latter observation was interpreted as follows. At low current, stimulation affected only neurons within one minicolumn, resulting in a bias. At high current, stimulation also affected neurons in other minicolumns, with opposite preferred direction (Albright et al., 1984). This increased ambiguity and lead to higher choice variability. In the language adopted here, the increase in choice variability was due to noisier input signals.

Second, experiments in mice found that optogenetic inactivation of OFC increased choice variability, and that this effect was due to animals reverting to stereotyped behavior (Kuwabara et al., 2020). In other words, when OFC was inactive, mice "chose" by adopting strategies such as selecting the same juice chosen in the previous trial or consistently licking on one side. These results indicated that OFC is necessary for economic choices. However, the results did not disambiguate whether OFC's role is in value assignment, value comparison, or both.

Third, in our recent experiments, monkeys chose between two juices offered sequentially (Ballesta et al., 2020). Electrical stimulation was delivered during presentation of the first offer 
(offer 1 ) or the second offer (offer2) at 25,50 or $125 \mu \mathrm{A}$ (in separate sessions). We measured three behavioral effects: range-dependent bias, changes in order bias, and increase in choice variability. Both range-dependent bias and changes in order bias were interpreted as due to the stimulation altering offer values by increasing the activity of offer value cells (input signals). These biases were observed in different ways at all current levels $\geq 25 \mu \mathrm{A}$ (see Discussion). Most relevant here, stimulation at $125 \mu \mathrm{A}$ during offer2 but not during offer1 induced an increase in choice variability. Since the decision was made only upon presentation of offer2, it was tempting to interpret the last results as evidence that the stimulation interfered with value comparison and that this process took place within OFC. However, high current stimulation could affect fibers in the white matter adjacent to the stimulation site (Stoney et al., 1968) and thus disrupt transmission to or between other brain areas (Jensen and Durand, 2009). Since the increase in choice variability was measured only at $125 \mu \mathrm{A}$, one cannot exclude that the decision process took place in some other brain region and/or that stimulation disrupted the early stages of motor planning (Cai and Padoa-Schioppa, 2014).

Ideally, to provide evidence that OFC contributes causally to value comparison, one would need some condition in which weak electrical stimulation increases choice variability but does not induce any range-dependent bias or change in order bias. In other words, the stimulation should disrupt value comparison without altering offer values per se. In such a condition, the stimulation weakness would ensure that its effects are confined to OFC (Stoney et al., 1968), and the absence of spatial or motor signals in this area (Carmichael and Price, 1995; Padoa-Schioppa and Assad, 2006; Grattan and Glimcher, 2014) would ensure that the stimulation does not disrupt motor planning. Thus one could conclude that OFC participates in value comparison. In the experiments described below, we identified such a condition.

\section{Results}

\section{Weak electrical stimulation of OFC disrupts value comparison}

In each experimental session, a monkey chose between two juices labeled $A$ and $B$, with $A$ preferred, offered in variable amounts. The two offers were presented sequentially in the center of the monitor (Fig.1A). Trials in which juice A was offered first and trials in which juice $B$ was offered first were referred to as "AB trials" and "BA trials", respectively. The terms "offer1" and "offer2" referred to the first and second offer, independent of the juice type and amount. For each pair of juice quantities, the presentation order (AB, BA) and the spatial location of the saccade targets varied pseudo-randomly and were counterbalanced across trials. Sessions typically included $\sim 400$ trials and offered quantities varied from trial to trial pseudo-randomly. An "offer type" was defined by two juice quantities in given order (e.g., [1A, 3B] or [3B, 1A]). To discourage monkeys from making a decision prior to offer2, we designed offer types such that for most values of offer 1 the animal split choices between the two offers (Ballesta and PadoaSchioppa, 2019).

Weak electric current was delivered during offer1 or during offer2, in separate sessions. In each session, stimulation was delivered in half of non-forced choice trials, pseudo-randomly selected (see Methods). Compared to previous work using electrical stimulation of OFC (Ballesta et al., 2020), the main difference is that in the present study we used very low currents, in the range 5$15 \mu \mathrm{A}$. 
For each session, we examined separately trials with and without the stimulation (stimON, stimOFF). For each group of trials, we analyzed choices with a probit regression:

$$
\begin{aligned}
& \text { choice } B=\Phi(X) \\
& X=a_{0}+a_{1} \log \left(q_{B} / q_{A}\right)+a_{2}\left(\delta_{\text {order }, A B}-\delta_{\text {order }, B A}\right)
\end{aligned}
$$

where choice $B=1$ if the animal chose juice $B$ and 0 otherwise, $\Phi$ was the cumulative function of the standard normal distribution, $q_{A}$ and $q_{B}$ were the quantities of juices $A$ and $B$ offered, $\delta_{\text {order }, A B}=1$ in $A B$ trials and 0 in $B A$ trials, and $\delta_{\text {order }, B A}=1-\delta_{\text {order, } A B}$. From the fitted parameters, we derived measures for the relative value $\rho=\exp \left(-a_{0} / a_{1}\right)$, the sigmoid steepness $\eta=a_{1}$, and the order bias $\varepsilon=a_{2}$. Intuitively, $\rho$ was the quantity ratio $q_{B} / q_{A}$ that made the animal indifferent between the two juices, $\eta$ was inversely related to choice variability, and $\varepsilon$ quantified the order bias. Specifically, $\varepsilon<0(\varepsilon>0)$ indicated a bias in favor of offer1 (offer2).

Fig.1BC illustrates the main results of this study. In one example session (Fig.1B), weak stimulation $(5 \mu \mathrm{A})$ was delivered during offer1. The stimulation did not substantially alter the relative value $(\rho)$, the order bias $(\varepsilon)$, or the sigmoid steepness $(\eta)$. In another example session (Fig.1C), weak stimulation (5 $\mu \mathrm{A})$ was delivered during offer2. Again, the stimulation did not substantially alter the relative value $(\rho)$ or the order bias $(\varepsilon)$. However, electrical stimulation substantially decreased the sigmoid steepness $(\eta)$. In other words, weak stimulation during offer2 selectively increased choice variability.

Similar patterns were observed at the population level. Our data set included $N=49$ sessions in which weak electrical stimulation was delivered during offer 1 , and $\mathrm{N}=42$ sessions in which weak stimulation was delivered during offer2. In general, neither the relative value $(\rho)$ nor the order bias $(\varepsilon)$ were substantially altered by electrical stimulation in either time window

(Fig.2AB). In contrast, the sigmoid steepness $(\eta)$ was significantly reduced by stimulation delivered during offer2 but not during offer1 (Fig.2C). That is, weak electrical stimulation during offer2 but not during offer 1 significantly increased choice variability. Importantly, this effect was observed in each of two animals (Fig.S1).

\section{Weak stimulation of OFC does not induce reversion to stereotyped behavior}

We conducted several control analyses. First, we considered the possibility that the increase in choice variability measured at very low current might be driven by reduced motivation or by a generic disengagement from the task. Arguing against this view, electrical stimulation in either time window did not systematically increase the error rate (Fig.3A), nor did it alter response times (Fig.3B).

Second, previous work found that optogenetic inactivation of OFC in mice induced an increase in choice variability, and that this effect was due to animals reverting to stereotyped behavior (Kuwabara et al., 2020). We thus examined whether weak electrical stimulation of OFC in monkeys affected choices in similar ways. Other things equal, animals might have a choice bias favoring one side (side bias). Similarly, they might tend to choose on any given trial the same option chosen in the previous trial (choice hysteresis) (Padoa-Schioppa, 2013; Alos-Ferrer et al., 2016; Schoemann and Scherbaum, 2019). These biases - or stereotyped behaviors - would contribute to choice variability defined in Eq.1. Thus in a series of analyses, we examined whether electrical stimulation altered these biases. Of note, in our choice task, the tendency to 
repeat choices (choice hysteresis) could refer to the juice type, the target location or the presentation order. Using probit regressions, we considered each potential bias in turn.

For the side bias, we examined each group of trials (stimOFF, stimON) with the following model:

$$
\begin{aligned}
& \text { choice } B=\Phi(\mathrm{X}) \\
& \mathrm{X}=\mathrm{a}_{0}+\mathrm{a}_{1} \log \left(q_{B} / q_{A}\right)+\mathrm{a}_{2}\left(\delta_{\mathrm{A}, \text { right }}-\delta_{\mathrm{B}, \text { right }}\right)
\end{aligned}
$$

where $\delta_{\mathrm{J}, \text { right }}=1$ if the target associated with juice $\mathrm{J}$ was presented on the right and 0 otherwise, and $\mathrm{J}=\mathrm{A}, \mathrm{B}$. The side bias was defined as $\xi=\mathrm{a}_{2}$. A measure $\xi>0$ indicated that, other things equal, the animal tended to choose the left target. Population analyses showed that the side bias was not substantially altered by weak stimulation of OFC in either time window (Fig.4A). (If anything, stimulation during offer1 reduced the side bias.)

For choice hysteresis (juice type), we analyzed each group of trials (stimOFF, stimON) with the following model:

$$
\begin{aligned}
& \text { choice } B=\Phi(X) \\
& X=a_{0}+a_{1} \log \left(q_{B} / q_{A}\right)+a_{2}\left(\delta_{n-1, B}-\delta_{n-1, A}\right)
\end{aligned}
$$

where $\delta_{n-1, J}=1$ if in the previous trial the animal chose juice $\mathrm{J}$ and 0 otherwise, and $\mathrm{J}=\mathrm{A}, \mathrm{B}$. Choice hysteresis was quantified as $\theta_{\text {juice }}=a_{2}$. A measure of $\theta_{\text {juice }}>0$ indicated that, other things equal, the animal tended to choose the same juice chosen in the previous trial. Confirming previous reports (Padoa-Schioppa, 2013), a population analysis revealed a consistent choice hysteresis related to the juice type $\left(\right.$ mean $\left(\theta_{\text {juice }}\right)>0$ ). Critically, weak stimulation of OFC in either time window did not systematically alter this phenomenon (Fig.4B).

For choice hysteresis (side), we used the following model:

$$
\begin{aligned}
& \text { choice } B=\Phi(\mathrm{X}) \\
& \mathrm{X}=\mathrm{a}_{0}+\mathrm{a}_{1} \log \left(q_{B} / q_{A}\right)+\mathrm{a}_{2}\left(\delta_{\mathrm{n}-1, \text { side } \mathrm{B}}-\delta_{\mathrm{n}-1, \text { side } \mathrm{A}}\right)
\end{aligned}
$$

where $\delta_{n-1, \text { side } J}=1$ if the target associated with juice $J$ was in the same spatial position as that chosen in the previous trial and 0 otherwise, and $\mathrm{J}=\mathrm{A}, \mathrm{B}$. Choice hysteresis was quantified as $\theta_{\text {side }}=a_{2}$. A measure of $\theta_{\text {side }}>0$ indicated that, other things equal, the animal tended to choose the same saccade target as that chosen in the previous trial. Population analyses showed that weak stimulation of OFC in either time window did not systematically alter choice hysteresis related to the chosen side (Fig.4C).

Finally, for choice hysteresis (order), we used the following model:

$$
\begin{aligned}
& \text { choice } B=\Phi(X) \\
& X=a_{0}+a_{1} \log \left(q_{B} / q_{A}\right)+a_{2}\left(\delta_{n-1, \operatorname{order} B}-\delta_{n-1, \operatorname{order} A}\right)
\end{aligned}
$$

where $\delta_{n-1, \text { order } J}=1$ if the order of presentation of juice $J$ in the present trial was same of the juice chosen in the previous trial and 0 otherwise, and $\mathrm{J}=\mathrm{A}, \mathrm{B}$. Choice hysteresis was quantified as $\theta_{\text {order }}=a_{2}$. A measure of $\theta_{\text {order }}>0$ indicated that, other things equal, the animal tended to repeat (as opposed to alternate) choices according to the presentation order. 
Population analyses showed that weak stimulation of OFC in either time window did not systematically alter choice hysteresis related to the presentation order (Fig.4D).

In conclusion, the drop in choice accuracy induced by weak electrical stimulation delivered during offer 2 was not due to the animals reverting to stereotyped behavior.

\section{Discussion}

Electrical stimulation may increase choice variability by interfering with value computation and/or with value comparison. In our choice task, value computation took place during presentation of both offer1 and offer2, while value comparison took place only during presentation of offer2. Two lines of evidence suggest that weak electrical stimulation selectively affected value comparison. First, choice variability increased only when the stimulation was delivered during offer2. Second, the stimulation did not induce any order or range-dependent bias (i.e., it did not affect valuation per se). In principle, electrical stimulation could also increase choice variability by reducing the animal's motivation or engagement in the task. However, the absence of any effect on error rates or reaction times argues against this hypothesis. Finally, stimulation could increase choice variability by disrupting the early stages of action planning. However, the primate OFC lacks spatial or motor representations (Padoa-Schioppa and Assad, 2006; Grattan and Glimcher, 2014) and stimulation at very low current is unlikely to affect neurons in other brain regions. In conclusion, our results indicate that neuronal activity in OFC contributes to value comparison.

In the present study, the increase in choice variability induced by weak stimulation of OFC was not due to the animals reverting to stereotyped behavior. In contrast, we previously observed that optogenetic inactivation of OFC in mice significantly increased stereotyped behavior (and thus increased choice variability) (Kuwabara et al., 2020). This apparent discrepancy may be interpreted considering that optogenetic inactivation, which was procured through excitation of inhibitory interneurons, had a dramatic effect on the activity of OFC neurons (as confirmed by simultaneous neuronal recordings). In essence, optogenetic inactivation shut down and thus "took offline" the target area. Absent the neural substrate normally devoted to the computation and comparison of offer values, animals' motor responses were presumably guided by alternative computations - i.e., stereotyped behaviors such as repeating on any given trial the same choice made in the previous trial. In this view, the mental processes guiding choices under optogenetic inactivation were qualitatively different from those taking place under normal conditions. In contrast, the weak electrical stimulation used here presumably had a mild effect on neuronal activity in OFC, inducing noise or degrading the quality of value comparison without completely preventing neuronal computations from taking place. Thus, the mental processes guiding animals' choices were noisier, but not fundamentally different from those taking place under normal conditions.

\section{Comparing the effects of OFC stimulation at different current levels}

It is interesting to contrast the results of weak electrical stimulation, described here, with those previously obtained with higher currents (Ballesta et al., 2020). As noted above, we quantified three behavioral effects: range-dependent bias, changes in order bias, and increase in choice variability. To compare the results obtained with different protocols, we normalized the effect sizes (see Methods). Inspection of Fig.S2 reveals that three effects varied with the current level in different ways. 
First, the range-dependent bias had a bell-shaped trend. All our measures are consistent with the understanding that electrical stimulation up to $\sim 50 \mu \mathrm{A}$ induced physiological increases in neuronal firing rates (Stoney et al., 1968; Tolias et al., 2005; Ethier et al., 2006; Histed et al., 2009). The tuning curves of offer value cells are linear and range adapting (Padoa-Schioppa, 2009; Conen and Padoa-Schioppa, 2019). Thus increasing firing rates is equivalent to increasing each offer value by a quantity proportional to the value range. Hence, stimulation induced a choice bias favoring the juice offered with the larger range. This effect increased with the current level until $50 \mu \mathrm{A}$. At higher currents $(\geq 100 \mu \mathrm{A})$, electrical stimulation affects cells in more complex ways such as inducing antidromic spikes that can collide with natural spikes (neuronal hijacking) (Griffin et al., 2011; Van Acker et al., 2013; Hussin et al., 2015). Consequently, the range-dependent bias disappeared (Ballesta et al., 2020).

Second, changes in the order bias grew with the current level. All the measures are consistent with the idea that this effect was driven by decelerating response functions (Arsiero et al., 2007; La Camera et al., 2008) and/or by neuronal hijacking at high currents (Ballesta et al., 2020).

Third, the increase in choice variability had a U-shaped trend: it was observed at very low current $(5-15 \mu \mathrm{A})$ and at the highest current $(125 \mu \mathrm{A})$, but not between the extremes (25 and 50 $\mu \mathrm{A})$. Notably, the effect size at weak current exceeded that measured at high current (Fig.S2). The U-shaped trend suggests that increases in choice variability at very low current and at high current were mediated by different cellular mechanisms. Low current stimulation is believed to primarily activate inhibitory interneurons, which are smaller, have higher firing rate, and thus have lower stimulation threshold compared to pyramidal cells (Cai et al., 2011). Notably, neuronal recordings in monkeys performing the same choice task used here indicated that decisions rely on circuit inhibition (Ballesta and Padoa-Schioppa, 2019). Furthermore, current computational models suggest that value comparison relies on a balance between recurrent excitation and pooled inhibition (Wang, 2002; Rustichini and Padoa-Schioppa, 2015; Pettine et al., 2021). In these models, increasing inhibition makes decisions more impulsive and thus less accurate (Wong and Wang, 2006). Thus we speculate that weak electrical stimulation $(\leq 15 \mu \mathrm{A})$ disproportionately increased inhibition and thus disrupted the excitation-inhibition balance, which specifically affected value comparison. Conversely, stimulation at intermediate currents (25-50 $\mu \mathrm{A})$, which presumably activated both pyramidal cells and interneurons, might have affected choices in other ways while preserving the excitation-inhibition balance. Finally, stimulation at high current $(125 \mu \mathrm{A})$ might have increased choice variability for several reasons, including by inducing antidromic spikes and/or by affecting fibers of passage. Future work should examine these hypotheses in more detail. Importantly, regardless of the specific cellular mechanisms through which weak electrical stimulation affects neuronal activity, our results indicate that neurons in the primate OFC participate in value comparison. 


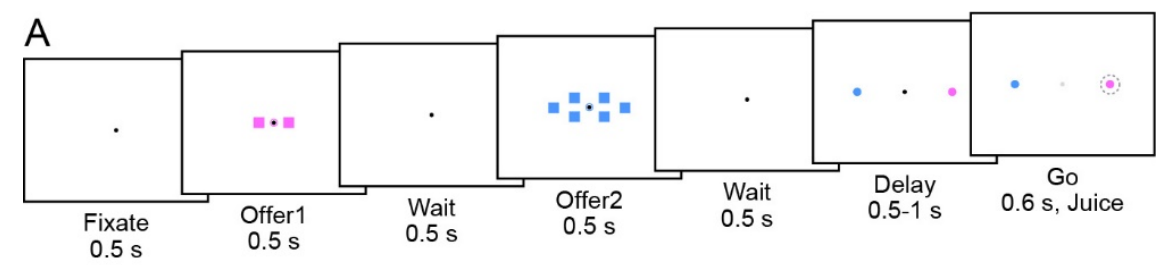

B

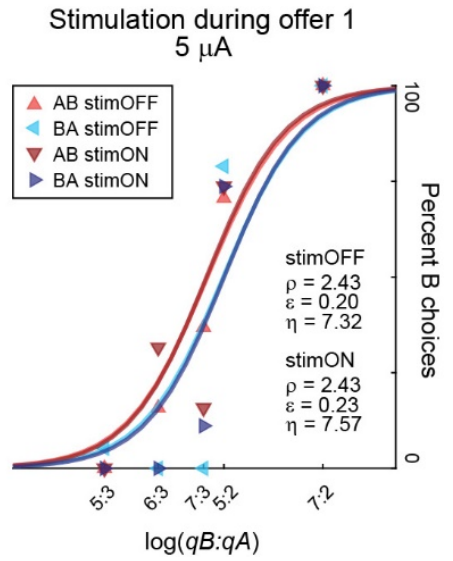

C Stimulation during offer 2 $5 \mu \mathrm{A}$

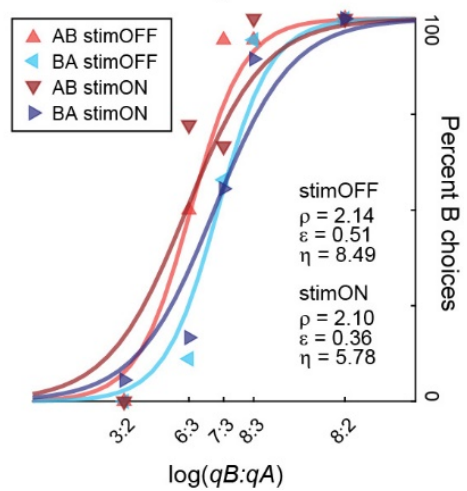

Figure 1. Experimental design and example sessions. A. Trial structure. Two offers, represented by sets of squares, appeared in sequence. Each trial began with the animal fixating a dot $\left(0.35^{\circ}\right.$ of visual angle). After a brief delay, two offers appeared in sequence, interleaved by a wait time. Each offer was represented by a set of colored squares (side $=1^{\circ}$ of visual angle); the color indicated the juice type and the number of squares indicated the juice amount. Along with the offer, a small colored circle $\left(0.75^{\circ}\right.$ of visual angle) appeared around the fixation dot. The circle indicated to the animal the juice identity in the case of null offer ( 0 drops; forced choices). The animal maintained center fixation throughout the trial until the go signal, indicated by the extinction of the fixation dot. The animal indicated its choice with a saccade and maintained peripheral fixation for an additional delay before juice delivery. Center fixation was imposed within $3^{\circ}$. Weak electrical stimulation (5-15 $\left.\mu \mathrm{A}\right)$ was delivered during offer 1 presentation or during offer 2 presentation, in separate sessions. BC. Example sessions. Each panel represents one session. Red and blue refer to $A B$ and $B A$ trials, respectively; light and dark colors refer to stimOFF and stimON, respectively. Sigmoids were obtained from probit regressions. For stimOFF trials and stimON trials, the order bias captured the distance between the red and blue sigmoids. Weak electrical stimulation was delivered during offer 1 ( $5 \mu \mathrm{A}$; panel A) or during offer2 ( $5 \mu \mathrm{A}$; panel B). Stimulation during offer 1 did not substantially alter any of the choice parameters $(\rho, \varepsilon, \eta)$. Similarly, stimulation during offer 2 did not substantially alter the relative value $(\rho)$ or the order bias $(\varepsilon)$. However, weak stimulation during offer 2 significantly decreased the sigmoid steepness $(\eta)$. 

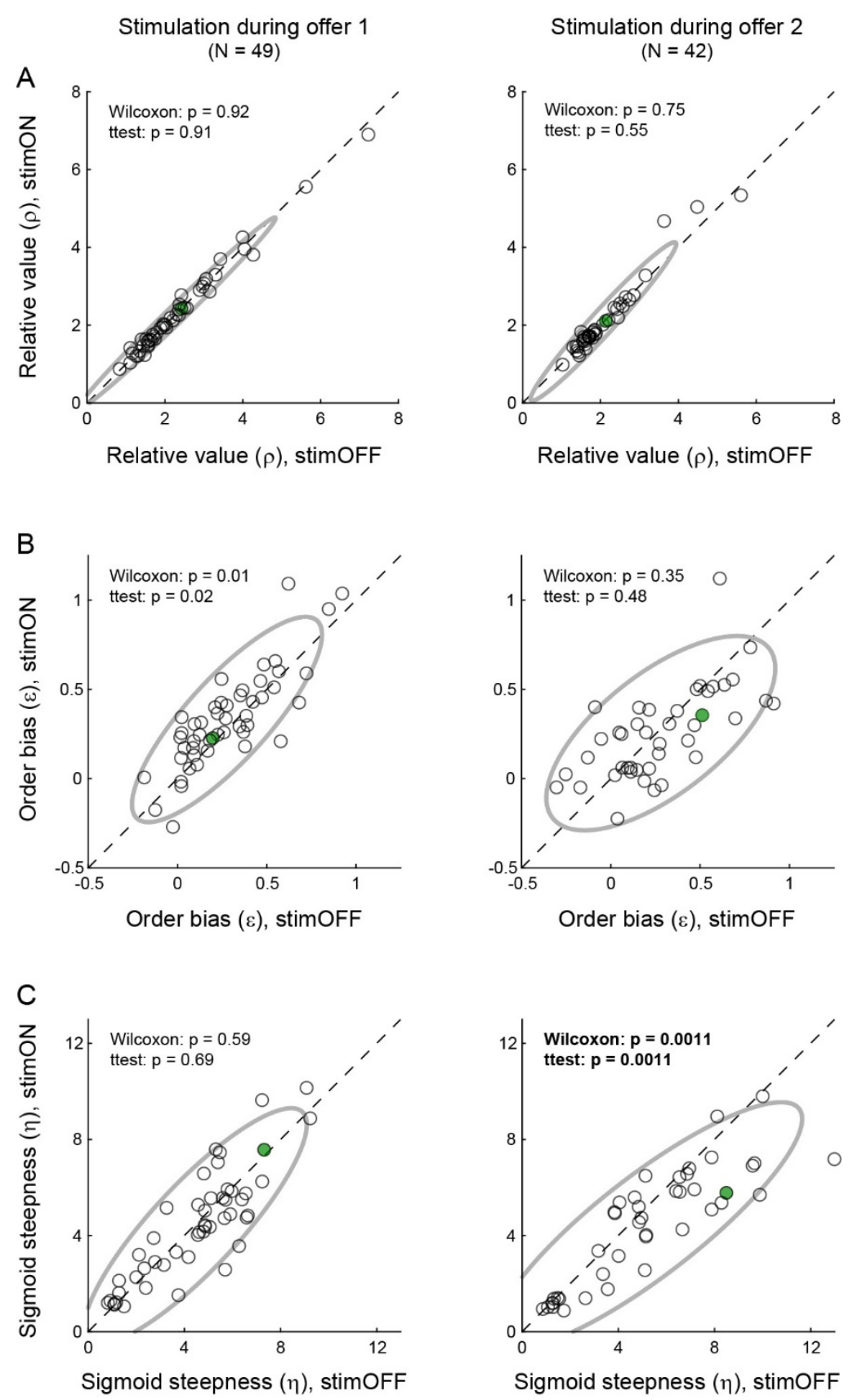

Figure 2. Weak stimulation of OFC selectively disrupts value comparison. A. Relative value $(\rho)$. The two panels illustrate the effects of stimulation during offer 1 and offer 2 , respectively. In each panel, $x$-axis and $y$-axis represent the relative value measured for stimOFF and stimON trials, respectively. Each data point represents one session and the ellipse represents the $90 \%$ confidence interval. Weak OFC stimulation during wither time window did not systematically alter the relative value. B. Order bias $(\varepsilon)$. Weak OFC stimulation during offer 2 presentation did not affect the order bias. Stimulation during offer 1 marginally increased this bias ( $p=0.02, t$ test). C. Sigmoid steepness $(\eta)$. Weak OFC stimulation during offer2 but not during offer1 induced a significant increase in choice variability. Each panel reports the $p$ values obtained from a two-tailed Wilcoxon test and a two-tailed t test. The two sessions illustrated in Fig.1BC are highlighted in green. 

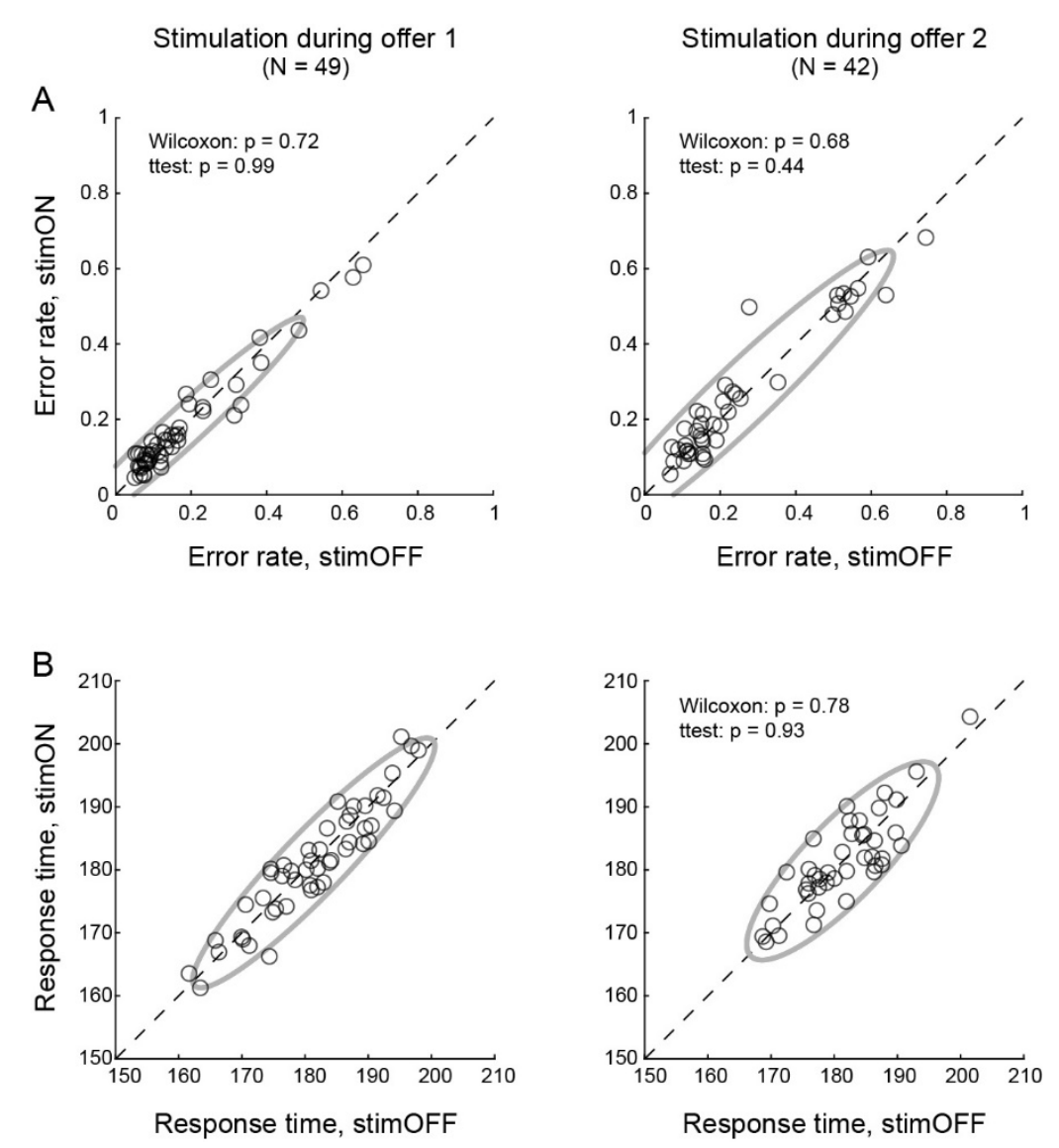

Figure 3. Weak stimulation of OFC does not affect error rates and response times. A. Error rate. Left and right panels illustrate the results obtained for stimulation at 5-15 $\mu$ A during offer 1 and offer2, respectively. In each panel, $x$-axis and $y$-axis represent the error rate measured for stimOFF and stimON trials, respectively. Each data point represents one session, the gray ellipse represents the $90 \%$ confidence interval, and the results of statistical tests are reported. Error rates were not significantly altered by stimulation in either time window. B. Response times. In each panel, $x$-axis and $y$-axis represent the mean response time measured for stimOFF and stimON trials, respectively. Other conventions are as in panel A. Response times were not significantly altered by stimulation in either time window. All $p$ values were obtained from two-tailed Wilcoxon tests and two-tailed t tests. 
bioRxiv preprint doi: https://doi.org/10.1101/2021.12.16.473020; this version posted December 19, 2021. The copyright holder for this preprint (which was not certified by peer review) is the author/funder, who has granted bioRxiv a license to display the preprint in perpetuity. It is made available under aCC-BY-NC-ND 4.0 International license.

Stimulation during offer 1 $(\mathrm{N}=49)$

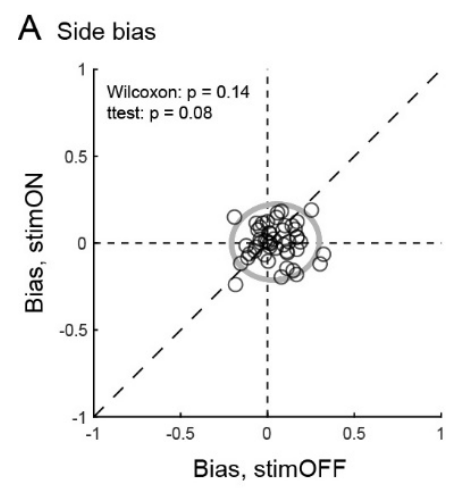

B Choice hysteresis: juice type

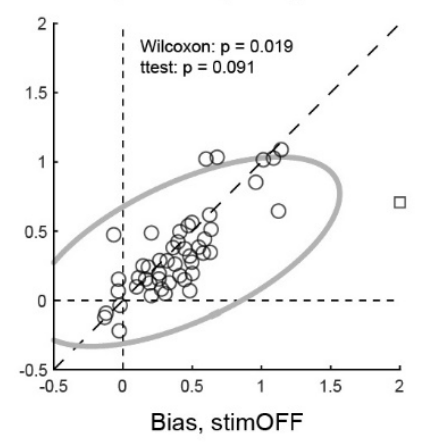

C Choice hysteresis: side

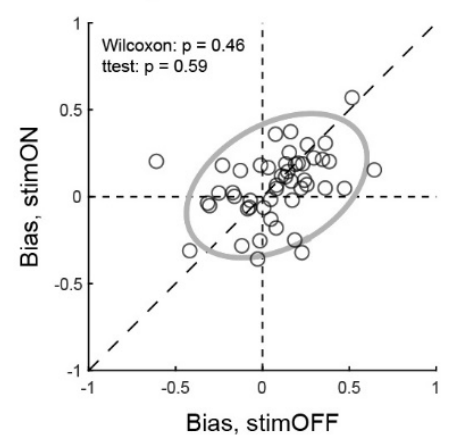

D Choice hysteresis: order

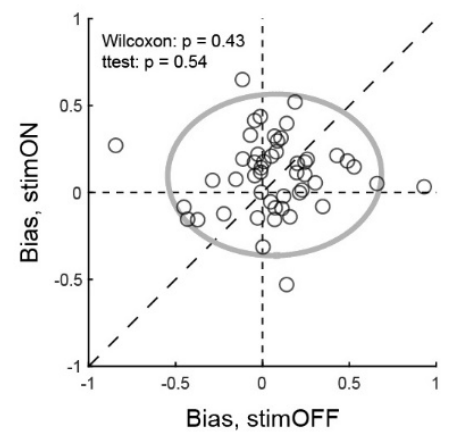

Stimulation during offer 2

$$
(\mathrm{N}=42)
$$
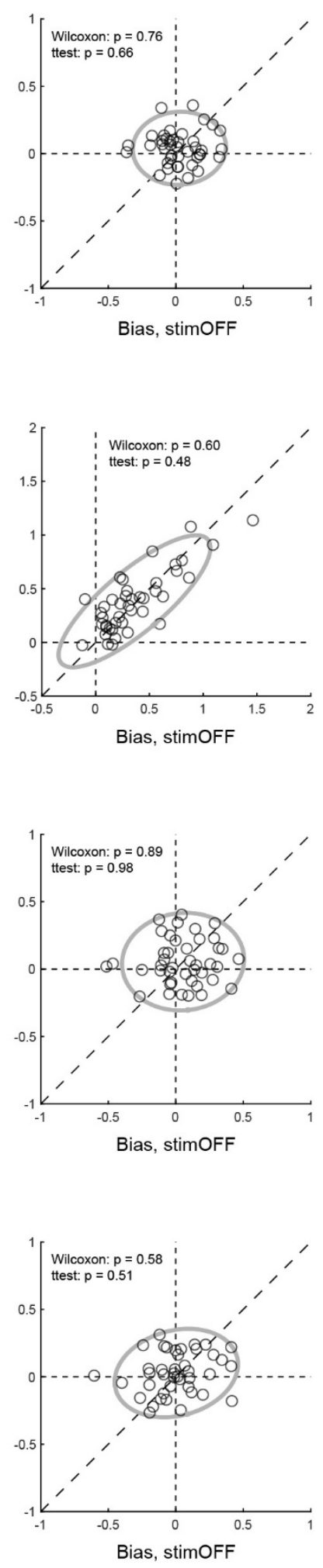

Figure 4. Weak stimulation of OFC does not increase stereotyped behaviors. A. Side bias. Left and right panel illustrate the results obtained for stimulation during offer 1 and offer 2 , respectively. In each panel, $x$-axis and $y$-axis represent the side bias measured for stimOFF and stimON trials, respectively. Each data point represents one session and the gray ellipse represents the $90 \%$ confidence interval. In essence, the side bias was very modest and electrical stimulation in either time window did not systematically increase it. B. Choice hysteresis, juice type. Same conventions as in panel A. As previously observed, monkeys generally showed significant choice hysteresis. However, this bias was not increased by electrical stimulation in either time window. The square symbol on the left panel represents one outlier $(y=3.28$; distance from mean $=5.11$ SD), which was removed from statistical analyses. CD. Choice hysteresis, side and order. Both biases were negligible and neither one was significantly altered by electrical stimulation in either time window. All $p$ values were obtained from two-tailed Wilcoxon tests and two-tailed $\mathrm{t}$ tests. 


\section{Methods}

All the experimental procedures conformed to the NIH Guide for the Care and Use of Laboratory Animals and were approved by the Institutional Animal Care and Use Committee (IACUC) at Washington University.

\section{Experimental procedures}

The study was conducted on two male rhesus monkeys (Macaca mulatta): G (age 8, $9.1 \mathrm{~kg}$ ) and $\mathrm{J}$ (age $7,10.0 \mathrm{~kg}$ ). Experimental procedures and data analyses closely resembled those previously described (Ballesta et al., 2020). Before training, we implanted in each monkey a head-restraining device and an oval recording chamber under general anesthesia. During the experiments, the animals sat in an electrically insulated enclosure with their head restrained. A computer monitor was placed $57 \mathrm{~cm}$ in front the animal. The behavioral task was controlled through custom-written software (http://www.monkeylogic.net/). The gaze direction was monitored by an infrared video camera (Eyelink; SR Research) at $1 \mathrm{kHz}$.

The chamber provided bilateral access to OFC. Structural MRIs ( $1 \mathrm{~mm}$ sections) performed before and after surgery were used to guide electrode penetrations. Electrical stimulation focused on the central orbital gyrus, in a region corresponding to area 13/11. During stimulation sessions, low-impedance (100-500 k 2 ) tungsten electrodes (100 $\mu \mathrm{m}$ shank diameter; FHC) were advanced using a custom-built motorized micro-drive driven remotely. The stimulating electrode was always positioned well within the gray matter. A second electrode advanced in parallel using the same micro-drive was used to confirm the depth and to record neuronal activity. Stimulation trains were generated by a programmable analog output (Power 1401, Cambridge Electronic Design) and triggered through a TTL by the computer running the behavioral task. Monopolar electric currents were generated by an analog stimulus isolator (Model 2200, A-M Systems).

Electric current was delivered during offer1 or during offer2, in separate sessions. For the present study, stimulation parameters were as follows. Stimulation started 0-100 ms after offer onset and lasted 300-600 ms. The stimulation train was constituted of biphasic pulses (200 $\mu \mathrm{s}$ each pulse, $100 \mu$ s separation between pulses) delivered at $125-200 \mathrm{~Hz}$ frequency. In different sessions, current amplitudes varied between 5 and $15 \mu \mathrm{A}$. Stimulation was performed in both hemispheres of monkey $\mathrm{G}$ (left: AP 31:36, ML -7:-12; right: AP 31:36, ML 4:9) and in both hemispheres of monkey J (left: AP 31:35, ML -8:-10; right AP 31:35, ML 6:10). Electric current was delivered unilaterally or bilaterally, in separate sessions. All the parameters were set at the beginning of each session and were not adjusted within sessions. Our data set included a total of 91 sessions ( 58 from monkey G, 33 from monkey J). Similar parameters were used for stimulation at higher currents ( $\geq 25 \mu \mathrm{A} ; 144$ sessions total) (Ballesta et al., 2020).

\section{Data analysis: Comparing effect sizes across current levels}

Data were analyzed in Matlab (MathWorks Inc). For each session, we examined separately trials with and without the stimulation (stimON, stimOFF). For each group of trials, we analyzed choices using probit regressions. From the fitted parameters, we derived measures for the relative value, the sigmoid steepness, and several choice biases (Eqs.1-5). A comparison of the behavioral effects of electrical stimulation at different current levels focused on three measures, namely the range-dependent bias, the change in order bias, and the increase in choice 
variability. Each effect was computed in each session, rectified such that the expected effect was $>0$, averaged across the relevant population, and normalized across conditions. The normalized effect sizes were thus defined as follows.

(1) Increase in choice variability. For each session, we measured the change in sigmoid

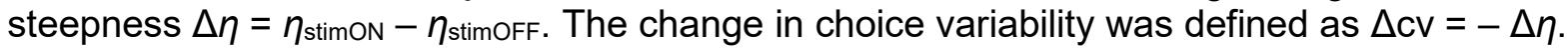
Thus $\Delta \mathrm{cv}>0$ indicated that the stimulation increased choice variability. For each time window (offer1, offer2) and for each current level $(\leq 15,25,50,125 \mu \mathrm{A})$, we averaged $\Delta \mathrm{cv}$ across the relevant sessions. We thus obtained a measure $\Delta \mathrm{cv}_{\text {condition }}$ for each of the 8 conditions. Finally, we divided these measures by the maximum, and obtained the normalized effect size shown in Fig.S2.

(2) Change in order bias. For each session, we measured the change in order bias induced by the stimulation $\Delta \varepsilon=\left(\varepsilon_{\text {stimON }}-\varepsilon_{\text {stimOFF }}\right)$. Suitable stimulation during offer1 (offer2) is expected to increase (decrease) the order bias. Thus we rectified the measures obtained for sessions where stimulation was delivered upon offer2 by changing the sign. For each time window (offer1, offer2) and for each current level $(\leq 15,25,50,125 \mu \mathrm{A}$ ), we averaged $\Delta \varepsilon$ across the relevant sessions. We thus obtained a measure for $\Delta \varepsilon_{\text {condition }}$ for each of the 8 conditions. Finally, we normalized these measures dividing by the maximum, and obtained the normalized effect size shown in Fig.S2.

(3) Range-dependent bias. Suitable electrical stimulation increases the firing rates of offer value cells and the effect is equivalent to increasing both offer values. Because of range adaptation, each offer value increases by a quantity proportional to the corresponding value range. As a result, the stimulation is expected to bias choices in favor of the juice offered with the larger value range. More precisely, indicating with $\Delta \mathrm{V}_{\mathrm{A}}$ and $\Delta \mathrm{V}_{\mathrm{B}}$ the value ranges for juices $A$ and $B$, respectively, the expected change in relative value $\Delta \rho=\rho_{\text {stimoN }}-\rho_{\text {stimOFF }}$ is proportional to the difference in value range $\Delta \mathrm{V}=\Delta \mathrm{V}_{\mathrm{A}}-\Delta \mathrm{V}_{\mathrm{B}}$ (Ballesta et al., 2020). We previously found that this effect was most pronounced at $50 \mu \mathrm{A}$ and did not depend on the stimulation window (Ballesta et al., 2020). To generate Fig.S2, we pooled sessions based on the current level $(\leq 15,25,50,125 \mu \mathrm{A})$. For each current level, the effect size was Pearson's correlation $\mathrm{r}(\Delta \rho, \Delta \mathrm{V})$ computed across sessions. The four measures were normalized dividing by the maximum effect size across current levels.

All the measures shown in Fig.S2 for current levels $\geq 25 \mu \mathrm{A}$ summarize results described in a previous study (Ballesta et al., 2020). Of note, here we use the label "125 $\mu A$ " instead of " $\geq 100$ $\mu \mathrm{A}$ " for clarity, because in the majority of these sessions (47/54) the current was set at $125 \mu \mathrm{A}$. In the remaining sessions (7/54), the current was set between 100 and $200 \mu \mathrm{A}$.

In each population analysis, we identified as outliers data points that differed from the mean by $>3$ STD on either axis, and we removed them from the data set. This criterion excluded 1/91 sessions at $\leq 15 \mu \mathrm{A}$ and $6 / 144$ sessions at $\geq 25 \mu \mathrm{A}$, as previously described (Ballesta et al., 2020), only from the analyses of range-dependent biases. Including these sessions in the analyses did not substantially alter any of the results. Exact $p$ values for these analyses are provided in Table S1.

\section{$\underline{\text { Data and code availability }}$}

The data set and the Matlab code used for the analysis are available upon request. 
Stimulation during offer 1

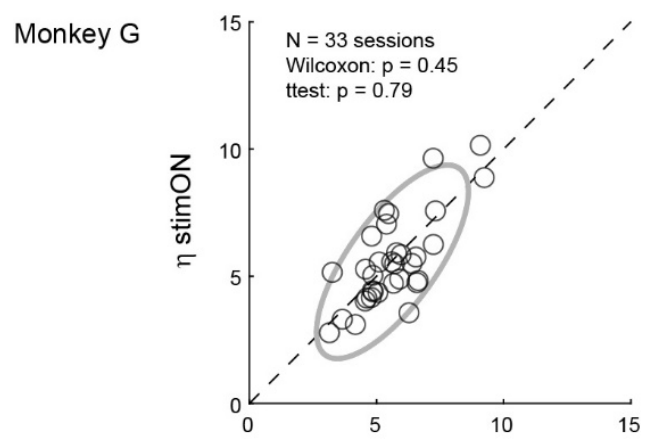

Monkey J

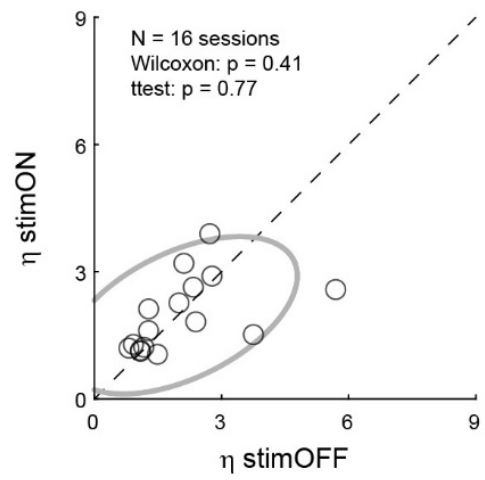

Stimulation during offer 2
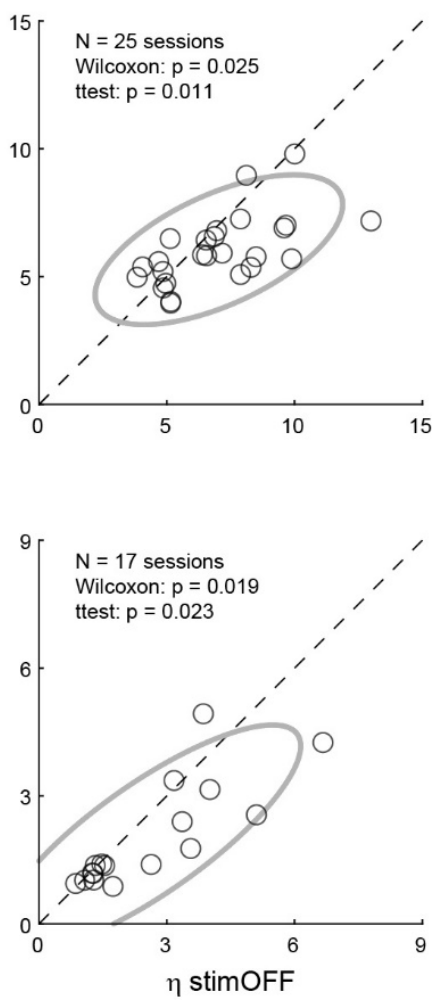

Figure S1. Effects of weak OFC stimulation on choice variability, individual animals. The figure summarizes the changes in choice variability measured upon stimulation at 5-15 $\mu$ A during offer 1 and offer2, for each animal. In each panel, $x$-axis and $y$-axis represent the sigmoid steepness measured for stimOFF and stimON trials, respectively. Each data point represents one session, the ellipse represents the $90 \%$ confidence interval, and results of statistical tests are reported. Stimulation during offer1 did not systematically alter the sigmoid steepness in either animal. In contrast stimulation during offer2 significantly reduced the sigmoid steepness in both animals. All $p$ values were obtained from two-tailed Wilcoxon tests and two-tailed t tests. 


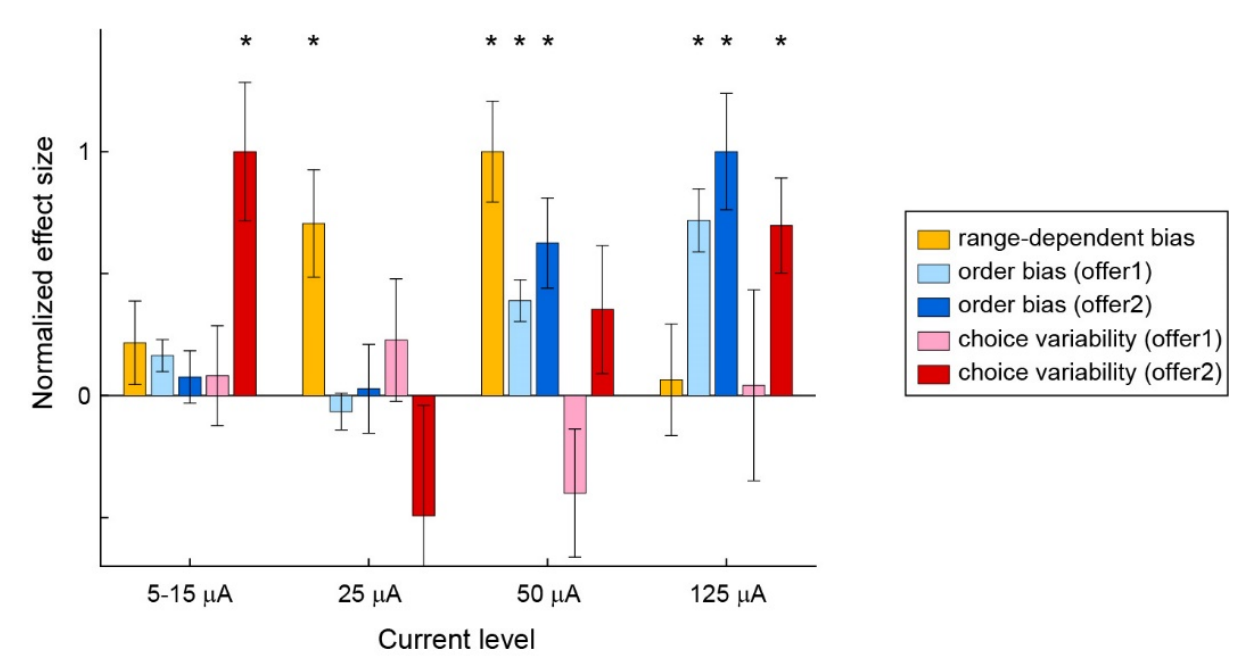

Figure S2. Normalized effect sizes measured at different current levels. Each effect was rectified and normalized across current levels such that the expected effect was $>0$ (see Methods). The rangedependent bias effect was defined as Pearson's correlation between the change in relative value $\left(\rho_{\text {stimon }}\right.$ $\left.-\rho_{\text {stimofF }}\right)$ and the difference in value range $\left(\Delta \mathrm{V}_{\mathrm{A}}-\Delta \mathrm{V}_{\mathrm{B}}\right)$. The change in order bias was defined such that the effect would be $>0$ if electrical stimulation in one time window biased choices in favor of the offer presented in the other time window. For choice variability, the effect was defined $>0$ if electrical stimulation increased choice variability. Effects were normalized such that the maximum across conditions $\mathbf{1}$ for each effect. Here histogram bars are population averages, error bars are standard errors, and asterisks indicate statistical significance $(p<0.01$; Pearson's correlation for range-dependent bias; two-tailed Wilcoxon test for all other effects). Exact $p$ values are provided in Table S1. For the range-dependent bias, sessions in which stimulation was delivered during offer 1 or offer 2 were pooled. Data at 5-15 $\mu \mathrm{A}$ are the same as in Fig.2. Data at 25, 50 and $125 \mu \mathrm{A}$ are from Ballesta et al. (2020). 
bioRxiv preprint doi: https://doi.org/10.1101/2021.12.16.473020; this version posted December 19, 2021. The copyright holder for this preprint (which was not certified by peer review) is the author/funder, who has granted bioRxiv a license to display the preprint in perpetuity. It is made available under aCC-BY-NC-ND 4.0 International license.

\begin{tabular}{|l|l|l|l|l|l|}
\hline & & $\mathbf{5 - 1 5} \boldsymbol{\mu A}$ & $\mathbf{2 5} \boldsymbol{\mu A}$ & $\mathbf{5 0} \boldsymbol{\mu A}$ & $\mathbf{1 2 5} \boldsymbol{\mu A}$ \\
\hline Range-dependent bias & N sessions & 90 & 46 & 40 & 52 \\
(offer1 + offer2) & mean \pm s.e. & $0.13 \pm 0.10$ & $0.70 \pm 0.22$ & $1.00 \pm 0.21$ & $0.06 \pm 0.23$ \\
& p value & 0.21 & $\mathbf{0 . 0 0 2 5}$ & $\mathbf{2 . 2 1 0 ^ { - 5 }}$ & 0.78 \\
\hline $\begin{array}{l}\text { Change in order bias, } \\
\text { offer1 }\end{array}$ & N sessions & 49 & 29 & 22 & 29 \\
& mean \pm s.e. & $0.05 \pm 0.02$ & $-0.07 \pm 0.08$ & $0.39 \pm 0.08$ & $0.72 \pm 0.13$ \\
& p value & 0.010 & 0.46 & $\mathbf{5 . 5 1 0 ^ { - 4 }}$ & $\mathbf{8 . 8 1 0 ^ { - 6 }}$ \\
\hline Change in order bias, & N sessions & 42 & 17 & 22 & 25 \\
offer2 & mean \pm s.e. & $-0.02 \pm 0.04$ & $0.03 \pm 0.18$ & $0.62 \pm 0.18$ & $1.00 \pm 0.24$ \\
& p value & 0.35 & 0.69 & $\mathbf{0 . 0 0 4 1}$ & $\mathbf{3 . 0 1 0 ^ { - 4 }}$ \\
\hline Change in choice & N sessions & 49 & 29 & 22 & 29 \\
variability, offer1 & mean \pm s.e. & $0.07 \pm 0.17$ & $0.22 \pm 0.24$ & $-0.39 \pm 0.26$ & $0.04 \pm 0.38$ \\
& p value & 0.59 & 0.47 & 0.20 & 0.84 \\
\hline Change in choice & N sessions & 42 & 17 & 22 & 25 \\
variability, offer2 & mean \pm s.e. & $0.82 \pm 0.23$ & $-0.48 \pm 0.44$ & $0.34 \pm 0.26$ & $0.68 \pm 0.19$ \\
& p value & $\mathbf{0 . 0 0 1 1}$ & 0.27 & 0.10 & $\mathbf{0 . 0 0 2 5}$ \\
\hline
\end{tabular}

Table S1. Normalized effect sizes and exact $p$ values across conditions. Mean and s.e. were rectified and normalized to the maximal effect across conditions (see Methods). For the range-dependent bias, $p$ values are from Pearson's correlation. All other $p$ values are from two-tailed Wilcoxon tests. Bold highlights $p$ values $<0.01$. 


\section{References}

Albright TD, Desimone R, Gross CG (1984) Columnar organization of directionally selective cells in visual area MT of the macaque. J Neurophysiol 51:16-31.

Alos-Ferrer C, Hugelschafer S, Li J (2016) Inertia and decision making. Front Psychol 7:169.

Arsiero M, Luscher HR, Lundstrom BN, Giugliano M (2007) The impact of input fluctuations on the frequency-current relationships of layer 5 pyramidal neurons in the rat medial prefrontal cortex. J Neurosci 27:3274-3284.

Bakkour A, Palombo DJ, Zylberberg A, Kang YH, Reid A, Verfaellie M, Shadlen MN, Shohamy D (2019) The hippocampus supports deliberation during value-based decisions. Elife 8

Ballesta S, Padoa-Schioppa C (2019) Economic decisions through circuit inhibition. Curr Biol 29:3814-3824 e3815.

Ballesta S, Shi W, Conen KE, Padoa-Schioppa C (2020) Values encoded in orbitofrontal cortex are causally related to economic choices. Nature 588:450-453.

Cai C, Ren Q, Desai NJ, Rizzo JF, 3rd, Fried SI (2011) Response variability to high rates of electric stimulation in retinal ganglion cells. J Neurophysiol 106:153-162.

Cai X, Padoa-Schioppa C (2014) Contributions of orbitofrontal and lateral prefrontal cortices to economic choice and the good-to-action transformation. Neuron 81:1140-1151.

Carmichael ST, Price JL (1995) Sensory and premotor connections of the orbital and medial prefrontal cortex of macaque monkeys. J Comp Neurol 363:642-664.

Cisek P (2012) Making decisions through a distributed consensus. Curr Opin Neurobiol 22:927936.

Conen KE, Padoa-Schioppa C (2019) Partial adaptation to the value range in the macaque orbitofrontal cortex. J Neurosci 39:3498-3513.

Ethier C, Brizzi L, Darling WG, Capaday C (2006) Linear summation of cat motor cortex outputs. J Neurosci 26:5574-5581.

Glimcher PW, Dorris MC, Bayer HM (2005) Physiological utility theory and the neuroeconomics of choice. Games Econ Behav 52:213-256.

Grattan LE, Glimcher PW (2014) Absence of spatial tuning in the orbitofrontal cortex. PLoS One 9:e112750.

Griffin DM, Hudson HM, Belhaj-Saif A, Cheney PD (2011) Hijacking cortical motor output with repetitive microstimulation. J Neurosci 31:13088-13096.

Hayden BY, Moreno-Bote R (2018) A neuronal theory of sequential economic choice. Brain and Neuroscience Advances:1-15. 
Histed MH, Bonin V, Reid RC (2009) Direct activation of sparse, distributed populations of cortical neurons by electrical microstimulation. Neuron 63:508-522.

Hunt LT, Hayden BY (2017) A distributed, hierarchical and recurrent framework for rewardbased choice. Nat Rev Neurosci 18:172-182.

Hussin AT, Boychuk JA, Brown AR, Pittman QJ, Teskey GC (2015) Intracortical microstimulation (ICMS) activates motor cortex layer 5 pyramidal neurons mainly transsynaptically. Brain stimulation 8:742-750.

Jensen AL, Durand DM (2009) High frequency stimulation can block axonal conduction. Exp Neurol 220:57-70.

Krajbich I, Armel C, Rangel A (2010) Visual fixations and the computation and comparison of value in simple choice. Nat Neurosci 13:1292-1298.

Kuwabara M, Kang N, Holy TE, Padoa-Schioppa C (2020) Neural mechanisms of economic choices in mice. Elife 9.

La Camera G, Giugliano M, Senn W, Fusi S (2008) The response of cortical neurons to in vivolike input current: theory and experiment : I. Noisy inputs with stationary statistics. Biol Cybern 99:279-301.

Murasugi CM, Salzman CD, Newsome WT (1993) Microstimulation in visual area MT: effects of varying pulse amplitude and frequency. J Neurosci 13:1719-1729.

Padoa-Schioppa C (2009) Range-adapting representation of economic value in the orbitofrontal cortex. J Neurosci 29:14004-14014.

Padoa-Schioppa C (2013) Neuronal origins of choice variability in economic decisions. Neuron 80:1322-1336.

Padoa-Schioppa C, Assad JA (2006) Neurons in orbitofrontal cortex encode economic value. Nature 441:223-226.

Pastor-Bernier A, Stasiak A, Schultz W (2019) Orbitofrontal signals for two-component choice options comply with indifference curves of revealed preference theory. Nat Commun 10:4885.

Pettine WW, Louie K, Murray JD, Wang XJ (2021) Excitatory-inhibitory tone shapes decision strategies in a hierarchical neural network model of multi-attribute choice. PLoS Comput Biol 17:e1008791.

Rich EL, Wallis JD (2016) Decoding subjective decisions from orbitofrontal cortex. Nat Neurosci 19:973-980.

Rustichini A, Padoa-Schioppa C (2015) A neuro-computational model of economic decisions. J Neurophysiol 114:1382-1398.

Salzman CD, Britten KH, Newsome WT (1990) Cortical microstimulation influences perceptual judgements of motion direction. Nature 346:174-177. 
Salzman CD, Murasugi CM, Britten KH, Newsome WT (1992) Microstimulation in visual area MT: effects on direction discrimination performance. J Neurosci 12:2331-2355.

Schoemann M, Scherbaum S (2019) Choice history bias in intertemporal choice. In. https://doi.org/10.31234/osf.io/7h9zi.

Song HF, Yang GR, Wang XJ (2017) Reward-based training of recurrent neural networks for cognitive and value-based tasks. In: Elife.

Stoney SD, Jr., Thompson WD, Asanuma H (1968) Excitation of pyramidal tract cells by intracortical microstimulation: effective extent of stimulating current. J Neurophysiol 31:659-669.

Tolias AS, Sultan F, Augath M, Oeltermann A, Tehovnik EJ, Schiller PH, Logothetis NK (2005) Mapping cortical activity elicited with electrical microstimulation using FMRI in the macaque. Neuron 48:901-911.

Van Acker GM, 3rd, Amundsen SL, Messamore WG, Zhang HY, Luchies CW, Kovac A, Cheney PD (2013) Effective intracortical microstimulation parameters applied to primary motor cortex for evoking forelimb movements to stable spatial end points. J Neurophysiol 110:1180-1189.

Wang XJ (2002) Probabilistic decision making by slow reverberation in cortical circuits. Neuron 36:955-968.

Wong KF, Wang XJ (2006) A recurrent network mechanism of time integration in perceptual decisions. J Neurosci 26:1314-1328.

Zhang Z, Cheng Z, Lin Z, Nie C, Yang T (2018) A neural network model for the orbitofrontal cortex and task space acquisition during reinforcement learning. PLoS Comput Biol 14:e1005925. 